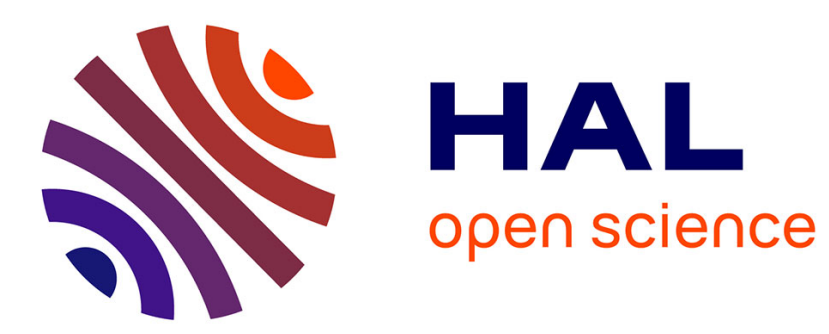

\title{
De la géographie féministe à la "gender geography": une lecture francophone d'un concept anglophone
}

Sophie Louargant

\section{To cite this version:}

Sophie Louargant. De la géographie féministe à la "gender geography ": une lecture francophone d'un concept anglophone. Espace Populations Sociétés, 2002, 3, pp.397-410. halshs-00261480

\section{HAL Id: halshs-00261480 \\ https://shs.hal.science/halshs-00261480}

Submitted on 23 Mar 2008

HAL is a multi-disciplinary open access archive for the deposit and dissemination of scientific research documents, whether they are published or not. The documents may come from teaching and research institutions in France or abroad, or from public or private research centers.
L'archive ouverte pluridisciplinaire HAL, est destinée au dépôt et à la diffusion de documents scientifiques de niveau recherche, publiés ou non, émanant des établissements d'enseignement et de recherche français ou étrangers, des laboratoires publics ou privés. 


\section{De la géographie féministe à la " gender geography » : une lecture francophone d'un concept anglophone From feminist geography to "gender geography": a French interpretation of an Anglo-Saxon concept.}

Mots clés : genre, concept, géographes, espace, territoire

Résumé français : Les écrits géographiques français sur le genre sont éparses et reflètent peu l'ambiguitté inhérente à ce concept : structurer une pensée dans un contexte culturel diffèrent. Le biais majeur serait de transposer les idées sans s'adapter aux singularités. Pour y parvenir l'une des vois possible est d'explorer la revue de littérature anglo-saxonne, de l'analyser pour cerner les enjeux et les limites des travaux. Des écrits émergent trois positionnements et perceptions de l'espace. Les frontières entre sociologie, ethnologie et géographie sont délicates à établir. L'article se propose de mettre en perspective ses points de vue.

Résumé anglais

Key words : gender, concepts, geographers, space, place

Abstract : The writings of French geographers about gender are scarce and do not really reflect the ambiguity inherent in this concept, that is to say organize a thought process according to various cultural backgrounds. There is a great temptation to dodge the issue and transform the ideas without considering singularities. One possible answer is to explore the literature review from English-speaking countries, to assess the issues at stake and the limits of the notions involved. Three positions and space perceptions emerge from these writings. The boundaries between sociology, ethnology and geography are unsure. The purpose of this paper is thus to take a close look at these viewpoints.

\section{Lire l'espace par le « genre » : un réel positionnement géographique ?}

Le présent article est d'abord et avant tout une mise en perspective de textes scientifiques anglosaxons positionnant le concept de genre ${ }^{1}$ dans divers champs d'application géographique. La revue de littérature réalisée se situe dans un contexte, celui d'un doctorat ${ }^{2}$ et se positionne dans le cadre de la géographie sociale et culturelle. Elle ne se veut pas une lecture exhaustive de tous les courants, mais tend à émettre une modeste catégorisation des géographes référents sur le domaine. Les analyses ne se résument donc pas à une traduction littérale, se veulent une lecture possible du concept. Ces réflexions possèdent un enjeu puisqu'elles s'inscrivent dans l'actuelle tendance des géographes déterminés à expliciter les " géographies anglo-saxonnes ${ }^{3}$ et amorcent les prémisses d'une réelle réflexion critique géographique en France.

Les études de genre prennent leurs origines dans le féminisme, donc dans un mouvement social plus focalisé sur les problèmes de domination et d'exclusion. S'inscrivant dans le cadre général des « women's studies », les écoles nord-américaines ont valorisé des revendications sociétales. Cette

\footnotetext{
${ }^{1}$ Le choix de l'utilisation du terme genre est délibéré, puisqu'il renvoie à la construction sociale des sexes, le terme " sexe » est de l'ordre du biologique et n'explicite pas la production, la reproduction des rôles, des représentations hommes-femmes, de l'ordre du culturel.

${ }^{2}$ Doctorat de géographie, Une lecture du territoire par le genre : analyse des trajectoires socio-spatiales dans l'agro-tourisme. Etude comparée sur trois terrains d'études : l'Ardèche méridionale, la région de Fès-Boulemane, la Ligurie (direction Hervé Gumuchian)

${ }^{3}$ CF.L'ouvrage collectif de J.F. Staszack, B. Collignon, C. Chivallon, B. Debarbieux, I. Géneau de Lamarlière, C., 2001, Géographies anglo-saxonnes, Tendances contemporaines, éd.Belin, coll. Mappemonde, 313p.
} 
institution de faits politisés, du domaine de la contestation dans les sphères de la recherche, suscite encore à l'heure actuelle ${ }^{4}$ pléthores de discussions chez les géographes. La question des genres et/ou des sexes en géographie a été fondatrice d'un nouveau mode de pensée sur l'espace. En effet, le contexte d'émergence des travaux de recherche issus des mouvances constructivistes des années 1960 et 1970 ont remis en cause les moyens d'objectivation des savoirs scientifiques. La reconnaissance des courants féministes se situe dans l'étude d'une société perçue comme un système de rapports entre les "genres" (« sex/gender system »). Le « sexe social », étant de fait vu comme un objet et comme le résultat d'une idéologie politique ou spatiale a été intégré dans l'environnement des sciences humaines, ce que souligne Joan Kelly, 1979 : " to articulate a new scientific paradigm, a paradigm where the category of gender and the sex/gender system represent integral parts of scientific research into human society and its reality $»$.

L'émergence de conceptualisations anglo-saxonnes d'après un corpus d'idées politisantes a peu trouvé de répondants en France du fait de la croyance dans les valeurs universalistes et républicaines : « croyance intimement liée à la confiance accordée à l'universalité des connaissances scientifiques ${ }^{5} »$. Les féministes ont progressivement adopté des approches constructivistes sur le genre pour contester l'essentialisme. Les recherches menées se sont cloisonnées à regarder la place des femmes et des hommes dans les différentes sphères d'activités (marché de l'emploi). Ce principe est actuellement remis en cause, avec l'apport des gender studies, il a eu l'avantage de décrypter les concepts classiques de géographie en explicitant les transcriptions spatiales des systèmes de relations hiérarchisées entre hommes et femmes. Les apports des travaux post-modernes tendent à relater les imaginaires produits par les femmes. Ces trois tendances ${ }^{6}$ ont marqué la structuration de la recherche anglo-saxonne et constituent le fil conducteur de la note de lecture.

\section{L'émergence et la validation du concept de " genre " en géographie : des revendications politisées à l'objectivation des savoirs féminins}

Les apports des women's studies ont été initiés par des géographes quantitativistes avec les écoles de l'analyse spatiale et se sont attachés à démontrer les espaces de domination d'après le modèle patriarcal. Ce positionnement constitue encore de nos jours une des fondements des approches de genre en particulier dans le domaine du développement comme le propose Jeanne Basilliat et Christine Verschurur à propos des agricultrices africaines ${ }^{7}$ : « le système d'oppression patriarcale de leurs propres sociétés fut renforcé par le nôtre ; un double système de pouvoir masculin se mit en place accentuant la subordination et la marginalisation économique des femmes avec leurs enfants ». Les géographes ont retranscrit au niveau spatial cette configuration.

\subsection{Déconstruire les connaissances masculines : «l'espace est une construction patriarcal »}

Lorsque la contestation politique arrive à émerger dans le domaine scientifique, une cohorte de craintes s'en accompagnent. En effet, qu'attendre d'un courant de pensée qui aurait pour base des revendications plus que de réels concepts, remettant en cause les présupposés universalistes, les concepts d'espaces-temps, de culture et de territoire? Le cheminement proposé ici, est de démontrer l'évolution des notions.

\footnotetext{
${ }^{4}$ Cf- congrès de l'Association des Géographes Américains-New-York-février 2001-table ronde de Linda Mac Dowell.

${ }^{5}$ Gardey D, Löwy I. , 2000, L'invention du naturel, Les sciences et la fabrication du féminin et du masculin, Histoire des sciences, des techniques et de la médecine, Ed. des archives contemporaines, 227p.

${ }^{6}$ Louargant S., 1999, Les femmes et leurs trajectoires socio-spatiales : des temporalités pour un territoire du "genre". Etude de cas dans deux arrière-pays méditerranéens touristiques : I'Ardèche du Sud et le Rif marocain, mémoire de D.E.A., Institut de Géographie Alpine, Université Joseph Fourier, Grenoble 1, p.10- rejoignant également le positionnement de L.Bondi, M.Domosh, op.cit, pp.65

7 Jeanne Bisilliat, Christine Verschuur, 2000, Le genre : un outil nécessaire, introduction à une problématique, Paris : L'Harmattan, p.13
} 
Distinction entre la place des femmes à l'université et les études impliquant le concept patriarcal.

En premier lieu, le mouvement des féministes géographes, souvent inspiré des travaux sur la révolution scientifique de Thomas S. Khun ${ }^{8}$ et de Michel Foucault, décrivent la puissance masculine des structures en place. Cette initiative venue des women's studies de Berkley, s'est imposée pour les géographes comme le précise Joan Kelly ${ }^{9}$ : "what we (feminists) are doing, is comparable to Copernicus shattering our geo-centricity, Darwin shattering andro-centricity, and the change is a fundamental, as dangerous, as exciting. And we are surprised that we are not welcome in the academy ? ". Pour ces géographes, inspirés des écrits philosophiques, il s'agissait de créer un nouveau paradigme qui permettrait à la géographie de s'orienter vers l'étude des " minorités » en évitant de s'exposer à une conception marginale sans fondement théorique et dans le court terme. Sandra Harding ${ }^{10}$, philosophe américaine, a posé les termes composants les théories féministes. Elle explique dans son ouvrage sur l'épistémologie et les perspectives féministes les fonctions de la connaissance scientifique masculine. Les systèmes de production et de reproduction sont les résultats d'une domination et d'une idéologie masculine : « the question of scientific institutions as cultural and ideological institutions of domination $»$.

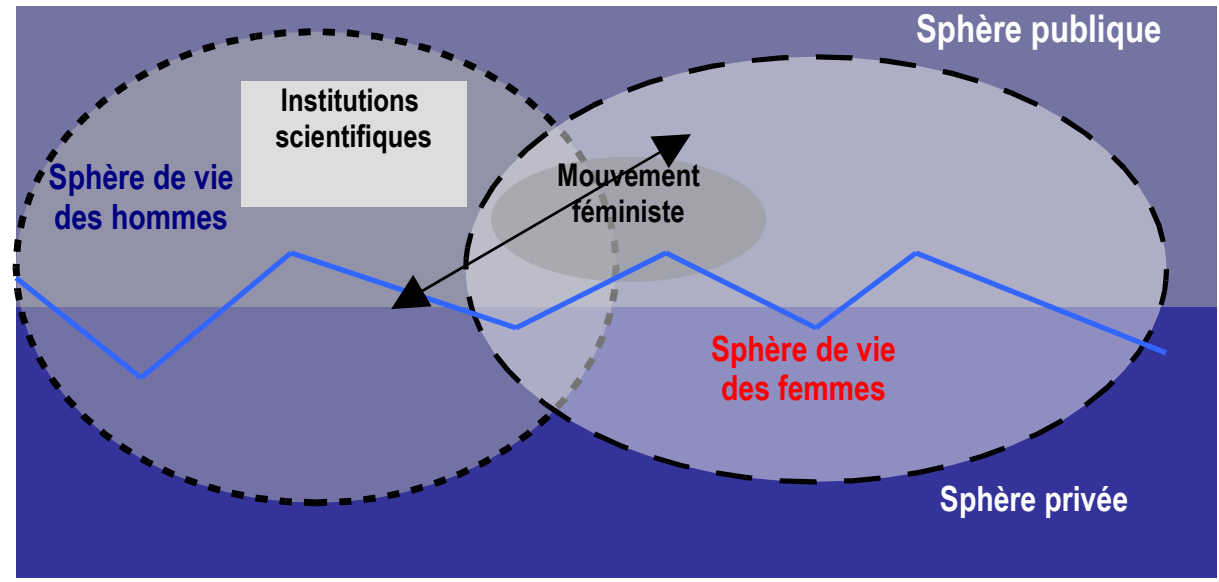

Figure $\mathrm{n}^{\circ} 1$ : Les sphères de vie et l'avènement du mouvement féministe comme une institution scientifique. D'après l'article de SAARINEN (A.), 1992, "Feminist research : in search of a new paradigm ?", p.126 La figure représente a sur-implication des hommes dans les sphères scientifiques

D'après l'objectif recherché, les premiers courants ont représenté un positionnement alternatif vis-àvis de la domination, du patriarcat. Cette étape décisive d'ouverture aux revendications exprimées par les féministes a favorisé l'élargissement du savoir des femmes et fut nécessaire pour introduire un nouvel ordre de production de la connaissance. L'aptitude plus ou moins opaque à répondre aux nouvelles exigences a privilégié dès le départ une étude en termes de classes sociales et assez catégorisante pour les individus. De ce fait, les courants féministes ont contribué à la production de rapports hommes ou femmes à l'espace très opposé, voire totalement distinct ignorant les connexions entre les deux individus. Cependant, dans les années 1980, la posture subjective de recherche est choisie. Elle propose un regard sur l'acteur social en tant que sujet individuel se positionnant dans le post-structuralisme (C.F. Figure $n^{\circ} 2$ ). Rose Gillian ${ }^{11}, 1993$, évoque cette marginalisation des travaux féminins : "women have been and continue to be marginalized as producers of geographical knowledge». Mettre en exergue cette différence de conceptualisation

\footnotetext{
${ }^{8}$ C'est-à-dire à tout ce qui touche des actes invisibles, des non dits, des nons formulées.

${ }^{9}$ SAARINEN (A.), 1992, Feminist research : in search of a new paradigm ?, pp.117 et KELLY (J.), 1984, "Women, History and Theory. The Essays of Joan Kelly", Chicago et Londre, The University of Chicago Press.

${ }^{10}$ HARDING (S.), MERRILL (B.), 1983, Discovering Reality. Feminist Perspectives on Epistemology, Metaphysics, Methodology and Philosophy of science, Dordrecht-Boston-Londre, édité par Reidel Publishing Company, pp. 311-324

${ }^{11}$ GILLIAN (R.), 1993, Feminism geography, The limits of geographical knowledge , 203p.
} 
équivaut, pour l'école d'Edeinburg, à dénoncer le patriarcat présent à l'université. Les féministes revendiquent une division de l'espace, du travail selon des principes de classes sexuées et s'engagent à défendre ces arguments au niveau politique et scientifique : " geography's epistemic exclusions are enacted by and impact on specific people »(op.cit.).

\subsection{Une vision radicaliste : «l'espace patriarcal produit des espaces dichotomiques »}

JAY (N.), féministe australienne ${ }^{12}$, propose de remonter aux fondements de la distinction des espaces: «logical dichotomy and radical gender distinctions as formally dichotomous, and some reasons why it is in the interest of certain social groups to understand gender distinctions in that way» Pourquoi les femmes ne peuvent-elles penser autrement, et comment peuvent-elles y arriver ? Pour démontrer la validité de la "gender-geography», les géographes posent les principales lignes directrices de leur démonstration d'après des éléments sociologiques. Le concept de " genre » se base sur les principes de la théorie des formes élémentaires d' Emile Durkheim ${ }^{13}$. II associe la dualité des sexes à la dualité du sacré et du profane. L'objectif de sa démarche causale est de déterminer les processus et les origines de construction de la dichotomie sociale (les lois de base des formes de pensée logique) existante. Les inégalités des sexes sont la caractéristique essentielle du paradigme féministe : inégalité des besoins des femmes (différents de ceux des hommes), inégalité d'espaces aménagés par des hommes traduisant une vision masculine excluant les spécificités féminines.

Dès le moment où les recherches ont abandonnée un cadre traditionnel des groupes sociaux pour considérer les femmes comme un "système " social intéressant, les réflexions sur l'espace social des femmes ont été critiquées. Gillian Rose précise que les femmes aussi sont des sujets d'étude : «in the face of such erasures and fantaisies, feminists have insisted that women are indeed subjetcs ${ }^{14}$ ».En tant qu'espace de socialisation, les femmes contribuent de manière différentes, canalisant d'autres processus de l'espace.

\subsection{Les fondements conceptuels: "les espaces dichotomiques révèlent des pratiques différenciées sexuées »}

Le reproche communément adressé aux géographes féministes est de s'inspirer trop fortement de concepts non-géographiques pour élaborer leurs hypothèses de recherche : "feminist geographers take heart from the examples of non-geographer feminists who use spatial language and metaphorical constructs as textual tools of empowerment" (op.cit., p. 245). "The feminist scholarship" conteste l'actuelle hégémonie académique des courants "gender" ou autres provenant de femmes "blanches", "hétérosexuelles" et occidentales, négligeant ainsi la variété des origines de femmes nonhétérosexuelles, non-blanches et du tiers-monde. Patricia Price-Chaltia ${ }^{15}, 1994$, associe cette identité au pouvoir de l'espace à la nécessaire connaissance à obtenir pour poser les postulats à développer : "knowledge and the power taht is bound up with knowledge is often constructed in spatial terms. Thus it comes as no surprise that feminists might choose the spatial as the site for contesting such relations of power". Ces espaces du pouvoir sont également l'expression d'une métaphore de la domination, une métaphore inculquée et cultivée par l'image matérielle de la femme. L'application aux études des pratiques spatiales a démontré des cheminements différenciés du quotidien (travaux de la "time-geography").

\footnotetext{
12 JAY (N.), Gender and Dichotomy, in "A reader in Feminist knowledge", éditée par Sneja Gunew, Londre et New York, pp. 89-105 ${ }_{13}^{13}$ DURKHEIM (E.), Les formes élémentaires, pp.40-41

${ }^{14}$ GILLIAN (R.), (?), Making space for the female subject of feminism, the spatial subversions of Holzer, Kruger and Sherman in Mappingthe subject, Geographies of cultural transformation, by Steve Pile and Nigel Thrift, parts 16

${ }^{15}$ PRICE-CHALTIA (P.),1994, Spatial metaphor and the politics of empowerment : mapping a place for feminism and postmodernism in geography ?, in Antipode, volume 26, $n^{\circ} 3, p .236-254$
} 

l'espace

\subsection{Le "genre" ou le sexe social : une définition transdisciplinaire}

Le concept de « gender » est apparu pour la première fois en 1972 dans l'ouvrage d'Ann Oakley ${ }^{16}$.La dimension sociale et culturelle de la différence des sexes qui dans la langue n'est pas exprimée, l'est dans cette notion. Elle démontre également la construction de modèles sociaux hommes et femmes révélateurs d'un processus de production de codification culturelle et non naturelle (biologique) prédestiné à l'homme et à la femme. Au Canada, il est présenté sous le terme de " sexo-spécificité » tandis que les féministes françaises le dénomme " catégories sociales de sexe ». Le sexe est alors pour ce courant de pensée une catégorie sociale. II l' est pour la distinction des femmes en tant que groupe et il est indivisible : " first, women do form a distinctive social group and, second, that the indivisibility of this group in traditional history is not to be ascribed to female nature ${ }^{17} »$.

L'évolution actuelle de la conjoncture des femmes dans le monde a entraîné à introduire la notion de " genre » dans les indicateurs statistiques. C'est ainsi que le Programme des Nations Unies pour le Développement Humain a introduit en 1995 deux critères pour mesurer la condition des femmes dans le monde : un indicateur sexospécifique (I.S.D.H.) ayant pour but de dégager les inégalités sociologiques et un indicateur de la participation des femmes (I.P.F.) à la vie économique et publique. Cette perception nouvelle de la place de la femme et l'utilité naissante accordée à la notion de « genre » par les instances internationales.

Ce champ de recherche a été exploré par les travaux ${ }^{18}$ de J.H. Momsen et concerne la géographie du développement, de la pauvreté et à démontrer la validé de construire des indicateurs sexués.

Les écrits anglo-saxons disponibles aujourd'hui font apparaître une voie de recherche principale : l'appropriation de l'espace par des individus et plus particulièrement les femmes. Elle résulte également de la possibilité d'envisager le territoire autrement d'après un autre regard, celui d'une différence de territorialité selon le « sexe social » d'un individu. Les phénomènes de spatialisation de l'espace du masculin au détriment de celui des féminins et esquivent les problèmes de territorialité (d'après la revue de littérature).

\subsection{Un retournement culturel : « les pratiques différenciées sont complémentaires »}

A l'origine, les féministes ont voulu détrôner la place de l'homme, vue comme un fondateur de l'organisation patriarcale du territoire. Pour ces géographes, la place de la femme est avant tout définie par les hommes. Ce principe se retrouve à chaque échelle du social, par contre c'est en terme de complémentarité que la définition de la relation de "genre » se construit. Si le " genre » se veut l'affirmation de la singularité de la femme dans une société pensée et crée par l'homme, le «nongenre » est alors l'affirmation de la singularité de l'homme. La relation de « genre » serait alors : la part des actions de la femme et de l'homme sur des espaces distincts mais complémentaires, un espace mixte viable uniquement par cette symbiose mais où il convient de cerner les interrelations tant dans un espace d'action que dans un espace du social dans un objectif précis : déterminer les lieux et les espaces de distinction, d'inégalité entre les sexes. L'interrelation entre un espace du domestique et du privée retranscrit l'échelle du « micro-socio-spatial » à l'échelle du « méso-sociospatial ». D'une échelle restreinte d'actions de l'homme et de la femme, où les individus possèdent leurs propres espaces, on procède à un passage vers une échelle supérieure, un espace où les individus agissent collectivement.

\footnotetext{
${ }^{16}$ Oakley Anne, Sex, Gender and Society, Temple Smith, Londres.

${ }^{17}$ "The Social Relation of the Sexes, Methodological Implications of Women's History », article sans références trouvé à l'université de Lund, réédition de l'article de la revue : Journal of women in Culture and Society, 1976, n 4 , pp. 809-823

${ }^{18}$ Momsen J. H. , Vivian Kinnaird, Different places, different voices, Gender and development in Africa, Asia and Latin America.
} 


\subsection{Un regard novateur : « la complémentarité crée des lieux de mixité »}

Doreen Massey s'est attachée à distinguer cette division, tout comme les premières études américaines issues des courants communautaires. S'y ajoutent des facteurs supplémentaires de contestations tel que la retranscription des habitudes existantes dans les sphères de la vie courante (privée et domestique) transposée à la production de connaissances. En effet, les courants les plus radicaux émettent l'hypothèse de repenser l'espace, d'après une production féminine du savoir géographique. Doreen Massey ${ }^{19}$ a formulé ce concept en exprimant une division du travail hommesfemmes dans les usines londoniennes. Ses recherches intégrant des éléments du spatial et du social, montrent l'existence d'interrelations entre les espaces sexués du local (lieu de travail à l'usine) pour les appliquer au niveau régional.

En effet, ils représentent les espaces d'échanges et de séparation des rôles entre l'homme et la femme. II apparaît que l'espace des hommes est un espace de séparation, qui pourtant unit autant qu'il sépare et dans lequel les femmes évoluent. Cependant, ces espaces de séparation possèdent des critères de mixité, de complémentarité, les travaux anglais sur le "tourisme sexuel" tendent à démontrer cette approche et valide le jeu complémentaire des transactions monétaires et des lieux de négociation hommes-femmes dans la prostitution.

\section{Les formes d'appropriation de l'espace en mouvement}

Les femmes et le pouvoir sont liés par un lien intrinsèque dans un espace mouvant, stable et inactif. La question sur laquelle se pencher est celle de savoir si l'espace macro-social des géographes dont on parle est différent de celui des géographes, pour dégager quels sont les critères d'identifications de cet espace du "genre". Comme Gillian Rose (1992) le propose c'est un espace double et paradoxal :"it's a space which is doubled, paradoxical, in which the ususal ways of thinking about location don't work." Par contre, d'après Evelyne J. Peters ${ }^{20}$, de nombreux géographes se sont attachés à déterminer comment les arrangements spatiaux sont impliqués dans la définition et la reproduction des groupes sociaux. Elle explique en quoi les groupes marginalisés vu comme des déviants ne peuvent s'intégrer dans l'espace. Elle se réfère à Robert Sack pour expliquer comment chaque individu construit le « nous », l'espace mixte dans lequel l'interprétation de chaque individu est lisible.("he argues that place can be used to construct "us" and "them", that place and space are linked to pratice through individuals recursive monitoring and interpretation of appropriate behaviour").

Doreen Massey distingue nettement cet apport des post-modernes: "sexism is an incredibly trenchant comonent spanning much of both modern and postmodern scholarship'. 1991. Cependant, la métaphore du pouvoir des hommes et des femmes est intimement lié aux actions politiques présentes sur un espace, l'absence de politique précise en son sein conduit à créer un « espace du négatif », symbolisant l'absence d'un lien, espace-femmes-politique: "The negative linking of woman, space and politics is made up of a two-step sequence. First, the female/feminine is conceptually equated with space and second, both are charged with an absence of politics or an inability to act politically. In sum, space=woman/women=apoliticism». ${ }^{21}$ Compte tenu des aspects mentionnés ci-dessus, n'est-il pas possible de concevoir un espace différent pour l'homme et la femme, sans pour autant mettre en exergue un espace du « négatif »?

\footnotetext{
${ }^{19}$ MASSEY (D.),1984, Spatial Divisions of labour, Social structures and the geography of production, 379 p./ 1995, « Space, place and gender $»($ ?)

${ }^{20}$ PETERS (E.J.), 1998, Subversive spaces : First Nations women and the city, in Environment and Planing D : Society and Space, volume ${ }^{\circ} 16$, pp. 665-685

${ }^{21}$ MASSEY (D.), 1995, Space, place and gender, p. 246
} 
II est important de reconnaître la production d'un espace, la différence d'une production et de reproduction de dichotomies spatiales. L'espace de la femme est de trois ordres : celui du travail domestique, celui du marché du travail, celui du travail de la communauté (Cf. Figure $\left.n^{\circ} 2\right)$. Ces trois espaces singuliers se juxtaposent, créent de fait un interstice: la " sphère » du sujet, c'est-à-dire l'élément micro-social d'expression de la singularité féminine.

Ici est présenté une troisième sphère, celle du sujet. Elle est l'une des ambitions de la " gendergeography ». L'espace en devenir, là où les éléments du social et du spatial se combinent pour exprimer la relation de " genre » revêt une importance déterminante si, de plus, l'on applique ces choix théoriques au domaine de la géographie de l'emploi.

Travail pour la « communauté »

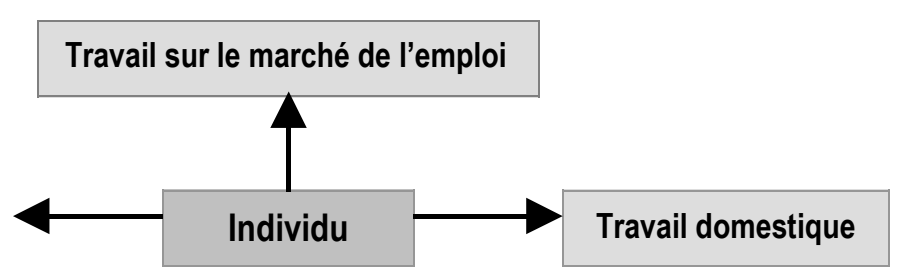

Figure $n^{\circ} 2$ : les sphères de travail. D'après MOORE MILROY (B.), WISMER (S.), 1994, "Communities, Work and Public/ Private Sphere Models", in Gender, Place and Culture, Volume 1, n`1, p.85

\subsection{L'articulation géographique : « les lieux mixtes sexués participent à la production de territorialités ॥}

Les travaux de Susan Hanson ${ }^{22}$ portent sur le rapport entre genre, travail et espace. Au travers de l'étude de l'évolution du travail des femmes dans le secteur industriel dans la région du Worcester (Massachussets), elle montre le lien entre spatialisation et trajectoires. Les lieux de " ghettoisation » montrent une tendance au " matriarcat » avec des dominations des femmes et de leurs activités dans les espaces de pouvoirs, considéré alors comme une «labor force ». L'objectif est ici de déterminer comment les frontières entre les hommes et les femmes sont construites dans différents quartiers (urbains, métropolitains et rural) et donc les rapports d'étanchéité : « the focus is on work, the segregation of men and women into different parts of the city[...] these differences are grounded and constitued in and trough space, place, and situated social networks ». Cette structuration engendre une occupation spatiale différenciée sexuée (sex-typing of occupation as a distance) visible dans la vie quotidienne. Ces travaux apportent une contribution aux recherches sur la segmentation du marché du travail et permettent d'être une contrebalance aux études contemporaines féministes. Ils ont mis en évidence d'une part la construction de formes urbaines spécifiques aux femmes. L'application méthodologique a été effectué sur les distances et les frictions entre les espaces publics et les espaces privés au niveau du marché du travail. L'intérêt a été de connecter l'étude sur différentes sphères et de voir " the friction of distance " au niveau cartographique. Les polarités public/privé, en liaison avec les aires métropolitaines, rurales ont été spatialisées. Le résultat significatif a démontré une différence de fréquentation (" gender gap ») d'après une journée médiane travail-domicile, dans différentes aires : « our finding about the differential friction of distance for

\footnotetext{
${ }^{22}$ Hanson S., Pratt G., 1995, Gender, work and space, Routledge, Londre et New York, 271p.
} 
women and men, and for different groups of women, prompts a second question that must be answered if we are to understand the implications of these patterns for people's access to jobs $\|^{23}$. Les travaux de Susan Hanson possèdent un caractère novateur puisqu'ils ont mis en exergue l'existences de places locales mixtes, le degré de variation d'accessibilité à ces lieux et l'importance accrue des réseaux sociaux en place. Les genres sont donc conditionnés par les structures de bases communautaires et renvoient à l'organisation de classes et à la structuration de la communauté locale( par exemple, le rôle des femmes impliquées dans les comités d'entreprises.

\section{La « gender rurality geography » : un nécessaire regard de "genre" en zone rurale}

Les distinctions d'espaces sont-elles applicables aux contextes socio-territoriaux existant à l'heure actuelle? Les problèmes rencontrés par les femmes sur un territoire rural doivent être examinés puisqu'ils sont des paramètres de construction. Watkins, $1995^{24}$, souligne un aspect déterminant à savoir, aider les femmes à sortir de leur rôle social encore très présent : " within the village there were few places for women to meet informally, so there was little opportunity to escape traditional gendered spaces and roles".

II convient également de dépasser les études micro-locales où la division sexuelle a déjà été démontré. Les représentations dominantes de la femme se traduisent selon l'occupation de trois lieux : l'espace domestique, l'espace d'aide dans l'exploitation, rôle "nourricier" (tâches domestiques, cuisine, allaitement...). L'espace du domestique est encore celui du féminin. L'approche de la "gender rurality " pose les principes d'une complémentarité selon les sexes et la nécessaire attention à distinguer les frontières opaques existantes entre les rôles afin de "casser »la dichotomie spatiale existante : " the role of women within rural communities should so be examined further to understand how certain women to maintain the moral boundaries of their community by perpetuating idealized representations of "the rural woman"25. Les apports réciproques des hommes et des femmes sur un espace sont souvent vus en termes d'obligations et de droits de chacun des deux sexes. Souvent, les femmes exerçant une activité sont encore perçue de manière négative par les autres femmes : "feminist geographers need to analyse how women can exclude and marginalize other women from communities by representing themselves as the "norm" vis-à-vis those who have an alternative culture or worldview» (op.cit).

A ce titre, la "gender rurality geography " n'a pas trouvé actuellement ses propres limites et frontières de recherche dans les études rurales: «to etasblished boundaries of gender research in rural studies » (op, cit,p.1). La difficulté des études de la relation de " genre » est due au mélange socio-culturel et économique d'un secteur local spécifique. Cependant, elle doit prendre en compte cette complexité pour constituer et comprendre les dynamiques sociales, spatiales en présence afin de trouver les identités, les valeurs des femmes en zones rurales :

"Evident here is a transition from thinking about gender in terms of men and women as fixed social categories, exhibiting behaviours which accord with established social norms (gender roles theories, Connel, 1987) to reflexive or interpretative, theories of the unstable and fragmented meanings and pratices attached to gendered subjects (gender identities theoies, Fraser 1989). The interests contesting rural restructuring build and, in turn, reshape gender relations; empowering women in differents ways in particular localities, complicated by their intersection with other axes of social power relations, notably class, "race" and ethnicity » (op.cit., p.2).

\footnotetext{
${ }^{23}$ Hanson S., Pratt G., 1995, Gender, work and space, Routledge, Londre et New York, p.105

${ }^{24}$ WATKINS F., 1995, The cultural Construction of Rurality : Gender Identities and the Rural Idyll, pp. 386-391

${ }^{25}$ WATHMORE (S.), MARDSEN (T.), LOWE (P.), 1994, Gender and rurality, David Fulton Publishers, London, $49 \mathrm{p}$.
} 
Les femmes agricultrices sont dans un système de relations sociales, familiales encore souvent basées sur la présence du patriarcat et ont du mal à poser les termes de ce que l'on nomme le " contrat sexuel », c'est-à-dire les bases d'échanges à l'intérieur des différents espaces. Toutefois, l'espace mixte d'expression de la relation du « genre » est un lieu de production de spatialités, une matrice d'intersection entre diverses individualités. Elizabeth Teather montre justement comment cette production de l'espace ne dépend pas uniquement d'une matrice mais relève d'une intersection complexe d'éléments créant de fait des séquences de spatialités quotidiennes «day-to-day».

A ce titre Linda Mac Dowell, qui étudie les relations entre hommes et des femmes dans l'espace pose en préambule de son ouvrage Gender Identity and place, understanding feminist geographies p.1), les questions récurentes aux études de genre : "où sont les hommes, où sont les femmes ? Comment les hommes et les femmes, vivent dans les diffèrentes parties du monde ? Et si les genres sont socialement construits, comment la féminité et la masculinité varient dans le temps et l'espace ?". Ces questions résument les choix majeurs des études de genre dans les courants postmodernes où le sujet du féminin se rèfère au sujet du masculin.

\section{Rapport au territoire et «au monde »: entre complémentarité des espaces et « connaissances situées » des lieux}

Le courant post-moderne s'attribue la signification de métaphore comme le souligne Liz Bondi, $1992^{26} . .$. « the prominence of spatial references in contemporary texts ... remains unexamined ... its exploration requires a different kind of "reading " from that usually practised wtihin geography : a kind of reading intent upon disclosing disruptive possibilities that remain latent in all texts. Again, feminism is a necessary partner in such a project, not least because of the intertwining of "woman" and "space" as figures in post-modern discourse".Certaines théoriciennes du "genre" établissent les fondements d'un "dualisme sexué" situant ainsi le problème des relations de "genre" à l'intérieur de la construction du pouvoir. Pouvoir souvent rattaché à un symbolisme puisé dans les écrits de la nativité quelque soit sa provenance ethnique ou religieuse, qui assigne un rôle, masculin et féminin, prédéterminé à chaque individu. Les systèmes d'alliance colportés par des récits sur la quintessence de la vie, sont enfouis dans un inconscient collectif.

\subsection{La validation de l'individu : « le rôle contextuel du sujet masculin ou féminin »}

Le débat porte sur la comparaison entre les politiques de revendication des théories féministes en géographie d'une part et sur les revendications plus globales de la société d'autre part. Les courants radicaux féministes, travaillant sur des minorités, qualifient souvent leur espace d'appropriation comme étant des lieux de périphérie, de marginalisation, et de "l'extérieur". On rentre dans la dichotomie négative de lieux dominants et de lieux dominés ("politically negative spatial terms"), rattachant ainsi la notion de "genre " à un conflit de pouvoir entre les hommes et les femmes et confèrent une connotation en tant que sujets.

\subsection{La confrontation masculin/féminin : « des subjectivités combinées »}

L'espace des femmes se situe plus par rapport à un espace construit avec l'autre et avec une périphérie sociale. L'homme, lui, est souvent représenté par rapport à lui-même, un centre qui s'autoconstruit. Cependant, le centre est souvent déterminé par la périphérie, du moins elle contribue à en établir les frontières. Les frontières entre le corps et l'esprit deviennent une métaphore spatiale pour exprimer ces inégalités spatiales et territoriales, et par là même, la relation de " genre ». II

\footnotetext{
${ }^{26}$ BONDI (L.), 1992, "Fragmens for geography ?", Antipode, n²4, p. 73-78
} 
existerait alors un interstice à investir par les femmes, un interstice dans lequel des femmes situent leur identité (les communautés lesbiennes, par exemple).

\subsection{La représentation de « soi »: « quand la géographie regarde les représentations sexuées corporelles »}

Le principe du concept du "female subject of feminism" est souvent en contradiction avec lui même : le sujet n'est pas "catégorisé ", pas divisé représentant à la fois le " sexe social », la race et les classes ("an identity made up of heterogenous and heteronomous representations of gender, race and class"). Or, ce concept chargé d'histoire est perçu comme une stratégie politique où le discours a une place déterminante("this notion of subjectivity as a political projet is political in various sense"). II réfute l'universalisme et accorde une cohérence à l'espace social si tant est qu'il soit porteur d'une logique dénonçant la discrimination "homme-femme ": "the effort to think through a subject position in terms of difference, contradiction and instability is connected to the effort to situate the production of knowledge, including knowledge about the subject, in a highly complex shifting and power-ridden world, and to render any action on the basis of knowledge both accountable to a specific position and vulnerable to other interpretations", Donna Haraway ${ }^{27}$. Basée sur l'existence d'une différence sexuelle, hiérarchisée, les géographes comme D.Domosh ou L. Bondi mettent en exergue l'existence de vérité, de connaissances situées en dehors du temps et du lieu.

A ce titre, les représentations des corps, de l'image de la femme dans l'art, la publicité sont au cœur des actuels travaux.

\section{Conclusion}

Dès trois courants présentés, le premier, plus politisé, se base sur un raisonnement dualiste, " marxiste », où les relations entre les individus et leur espace se posent en termes de classes d'hommes et de classes de femmes. Le second tend à déconstruire le premier et se positionne dans le post-modernism et le poststructuralisme afin d'étudier simultanément les hommes et les femmes pour cerner les interrelations existantes. Enfin, la troisième est une approche plus sociologique découlant de sociétés où « l'universel » de la condition humaine est prioritaire par rapport à la classification, en reconnaissant évidemment une spécificité féminine. Ces trois lignes de recherche sont le signe d'un positionnement en construction au sein de la discipline géographique.

L'appropriation et la maîtrise du temps de lieux par des femmes donne un sens restreint à l'espace contrairement à la vision masculine globale du lieu, dans lequel il s'intègrent parfaitement. Les femmes, elles, voient ce que font les autres produisent dans l'espace. Ces écrits mettent l'accent sur la notion temporelle, qui est l'un des éléments majeurs qui nous conduira à exploiter la notion de trajectoire des femmes

\footnotetext{
${ }^{27}$ Haraway D.,1991, "situated knowledges : the science question in feminism and the privilege of partial perspective", in D. Haraway Simians, Cyborgs, and women : the reinvention of Nature, New York : Routledge
} 


\section{Bibliographie}

Jeanne Bisilliat, Christine Verschuur, 2000, Le genre : un outil nécessaire, introduction à une problématique, Paris : L'Harmattan, 263p.

Bondi L., 1990, Feminism, postmodernism, and geography : space for women ?, in Antipode, n²2, pp.156-167

Bondi L., 1991, Progress in geography and gender : feminism and difference, Progress in Human Geography, $\mathrm{n}^{\circ} 14, \mathrm{pp} .438-445$

Bondi L., 1992, Gender and Dichotomy, in Progress in Human geography, n¹6, , pp.98-104

Bondi L., Domosh M., 2001, Autres figures en d'autres lieux : féminisme, postmodernisme et géographie in ouvrage collectif de J.F. Staszack, B. Collignon, C. Chivallon, B. Debarbieux, I. Géneau de Lamarlière, C., 2001, Géographies anglo-saxonnes, Tendances contemporaines, éd.Belin, coll. Mappemonde, pp.63-79

Bowlby S.R., Mackensie S., 1982, Feminism and geography, pp. 19- 21.

Chivallon C., 2001, Les géographies féministes, un plaidoyer convaincant de connaissances "situées", in ouvrage collectif de J.F. Staszack, B. Collignon, C. Chivallon, B. Debarbieux, I. Géneau de Lamarlière, C., 2001, Géographies anglo-saxonnes, Tendances contemporaines, éd.Belin, coll. Mappemonde, pp.57-63

Domosh M., 1988, Geography and gender : home, again ?, Progress in Human Geography, n²2, pp. 276-282

Domosh M., 1997, Geography and gender : the personal and the political, in Progress in Human Geography, $\mathrm{n}^{\circ} 21, \mathrm{pp} .81-87$

Duncan N., 1996, Body space. Destabilizing geographies of gender and sexuality, London and New York: Routledge, 433p.

Gardey D, Löwy I. , 2000, L'invention du naturel, Les sciences et la fabrication du féminin et du masculin, Histoire des sciences, des techniques et de la médecine, Ed. des archives contemporaines, 227p.

Halford S., 1992, Feminist change in a patriarchal institution : the experience of women's initiatives in local government, in M. Savage and A. Witz ('eds.), gender and bureaucracy., pages

Hanson, S., 1992, Geography and feminism: worlds in collision, Annals of the Association of American Geographers, volume 82, pp.569-86

Hanson S., Pratt G., 1995, Gender, work and space, Routledge, Londre et New York, 271p.

Hanson S., 1988, Spatial dimensions of the gender division of labour in a local labor market, Urban Geography, volume 9, pp.180-202

Harding S., Merrill B., 1983, Discovering Reality. Feminist Perspectives on Epistemology, Metaphysics, Methodology and Philosophy of science, DordrechtBoston-Londres, édité par Reidel Publishing Company, pp. 311-324

Haraway D.,1991, Simians, Cyborgs, and women : the reinvention of Nature, Routledge, Londres et New York, 255 p.

Jay N., 1981, Gender and Dichotomy, Feminist Studies n²: 38-56

Longhurst, R., 1995, The body and Geography, Gender, Place and culture, volume 2, pp. 97-105

Louargant S., 1999, Les femmes et leurs trajectoires socio-spatiales : des temporalités pour un territoire du "genre". Etude de cas dans deux arrière-pays méditerranéens touristiques : I'Ardèche du Sud et le Rif marocain, mémoire de D.E.A., Institut de Géographie Alpine, Université Joseph Fourier, Grenoble $1,163 \mathrm{p}$.

Mac Dowell L., 1999, Gender Identity and place, understanding feminist geographies, Polity Press, Cambridge et Oxford-, 284p.

Massey D., 1984, Spatial Divisions of labour, Social structures and the geography of production, Mac Millan, Londres, 379 p.

Massey D., 1994, Space, place and gender, Cambridge et Oxford-polity Press

Momsen J. H. , Vivian Kinnaird, Different places, different voices, Gender and development in Africa, Asia and Latin America.pages

Moore M., Wismer S., 1994, Communities, Work and public/private Sphere Models, Gender, Place and culture, volumen ${ }^{\circ}$, pp.71-87

Nicholson L.J. , 1990, Feminism/postmodernism, London, Routlege, 348p.

Pratt G. , 1993, Geographic metaphors in feminist theory, paper presented at "making worlds : metaphor and materiality in the production of feminist Texts Conference",October 14-16, Tuscon, Arizona. 
Price-Chaltia P.,1994, Spatial metaphor and the politics of empowerment : mapping a place for feminism and postmodernism in geography ?, in Antipode, volume $26, n^{\circ} 3$, pp.236-254

Rose G., 1993, Feminism and geography : the limits of geographical knowledge, Cambridge, Polity Press, 206p

Rose G., 1995, Distance, surface, elsewhere: a feminist critique of the space of phallocentric self/knowledge, in Environment and Planing D: Society and Space, volume 13, pp.761-781

Saarinen A., 1992, Feminist research : in search of a new paradigm ?, pp. 116-141

Spain D., 1992, Gendered spaces, chapel hill University of North Carolina Press, 256 p.

Wathmore S., Mardsen T., Lowe P., 1994, Feminist Perspectives in Rural Studies, David Fulton Publishers, London., 265 p.

Watkins F., 1995, The cultural Construction of Rurality : Gender Identities and the Rural Idyll, pp. 383-391 